\title{
Extension of a Formula by Cayley to Symmetric Determinants
}

\author{
By LaRs GäDING
}

(Received 25th November, 1946. Read 17th January, 1947.)

It has been proved by $\mathrm{C}_{A Y L E Y}$ that if $x_{11}, x_{12}, x_{21} \ldots$ are independent variables, $x=\operatorname{det}\left(x_{i k}\right), \xi=\operatorname{det}\left(\xi^{i k}\right),(i, k=1, \ldots n)$, where $\xi^{i k}=\partial / \partial x_{i k}$, then by formal derivation $\xi x^{a}=a(a+1) \ldots(a+n-1) x^{a-1}$. This is a special case of the formula ${ }^{1}$

$$
\xi^{i_{1}, i_{m} k_{1} . . k_{m}} x^{a}=a(a+1) \ldots(a+m-1) x^{a-1} x^{i_{1} . . i_{m} k_{1} \ldots k_{m}}
$$

where $\quad m=1, \ldots, n$ and $\xi^{i_{1} . .}=\operatorname{det}\left(\xi^{i k}\right)$ with $i=i_{1}, \ldots i_{m} ; k=k_{1}, \ldots k_{m}$ and $x^{i, \ldots}$ is the algebraical complement of $x_{i_{1} \ldots i_{n} k_{1} \ldots k_{m}}=\operatorname{det}\left(x_{i k}\right)$, $\left(i=i_{1}, \ldots i_{m} ; k=k_{1}, \ldots k_{m}\right)$, in $x=x_{1} \ldots n 1 \cdots n$.

In this note it will be shown that (1) holds also for symmetric determinants where $x_{i k}=x_{k i}$, provided that $\xi^{i k}=\frac{1}{2} \partial / \partial x_{i k}, \xi^{i i}=\partial / \partial x_{i i}$, $(i \neq k)$, and the factor on the righthand side is replaced by $a\left(\alpha+\frac{1}{2}\right) \ldots$ $\left(a+\frac{1}{2}(m-1)\right)$.

Let the sequences $i_{1} \ldots i_{m} i_{1^{\prime}} \ldots i_{m^{\prime}}$ and $k_{1} \ldots k_{m} k_{1^{\prime}} \ldots k_{m^{\prime}}$ be obtained from $1 \ldots n$ by even permutations. That is, $i_{1} \ldots i_{m}$ is a set of any $m$ of the first $n$ integers, while $i_{1^{\prime}} \ldots i_{m^{\prime}}$ is also such a set but not necessarily the same set. Expanding $x_{i}{ }^{\prime} \cdots i^{m^{\prime}} k_{1}^{\prime} \cdots k_{m^{\prime}}$ we have

$$
\begin{aligned}
& \sum_{p^{\prime}=1}^{m \prime}(-1)^{p^{\prime}} x_{i_{1}^{\prime}} k_{p^{\prime}} x_{i_{2}^{\prime}} \ldots k_{p^{\prime}-1} k_{p^{\prime}+1} \ldots \\
& =\Sigma_{p^{\prime}=1}^{m} x_{i_{1}} k_{p^{\prime}} x^{i_{1} \cdots i_{m^{2}} i_{1} k_{1}, k_{m^{k}} p^{\prime}} \text {. }
\end{aligned}
$$

Now write $\boldsymbol{i}=i_{1} \ldots i_{m}$ and $\boldsymbol{k}=k_{1} \ldots k_{m}$, put $x^{i \pi i k}=0$ if $i \in \boldsymbol{j}$ or $k \in \boldsymbol{k}$ and use the sum convention of tensor calculus, all sums running from 1 to $n$. The result is

$$
(n-m) x^{i k}=x_{i k} x^{i i k k}=x_{i k} x^{i j k \boldsymbol{k}}
$$

With $y_{i k}=x^{i k}$, Jacobi's formula gives $y_{i k}=x^{m-1} x^{i k}$ and $y^{i k}=x^{n-2} x_{i k}$ so that (2) gives the identity

$$
(n-m) y y_{i \mathbf{k}}=y^{i k} y_{i k \mathbf{k}} \text {. }
$$

The symmetry $x_{i k}=x_{k i}$ is used in

1 H. W. Turnbull, "The Theory of Determinants, Matrices, and Invariants," London, (1928), p. 116. 
LEMMA 1. Let $u \doteq i_{1} \ldots i_{m}$ and $u_{r}=i_{1} \ldots i_{r-\mathrm{J}} i_{r+1} \ldots i_{m}$ and analogously for $\boldsymbol{k}$ and $\boldsymbol{k}_{\text {.. }}$ Then

$$
m x_{i k}=\Sigma_{r, 8=1}^{m}(-1)^{r+\dot{s}} x_{k_{i} i_{r} i_{r} \boldsymbol{k}_{s}} .
$$

Expanding the righthand side, with $\boldsymbol{k}_{88^{\prime}}=\left(\boldsymbol{k}_{8}\right)_{s^{\prime}}$ written to denote the effect of suppressing both $k_{8}$ and $k_{z^{\prime}}$ from the set $k$, one gets

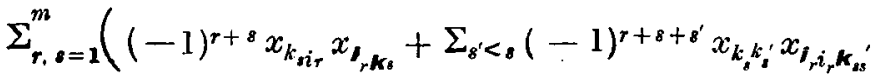

$$
\begin{aligned}
& \left.+\Sigma_{s^{\prime}>s}(-1)^{r+8+s^{\prime}+1} x_{k_{s k s^{\prime}}} x_{i, i, K_{s k^{\prime}}}\right)=m x_{i k} \\
& +\Sigma_{r} \Sigma_{s}>_{s}(-1)^{r+s+s^{\prime}}\left(x_{k_{s} k_{s}^{\prime}}-x_{k_{s} k_{s}}\right) x_{i_{r} i_{r} \boldsymbol{k}_{s s}}=m x_{i \boldsymbol{k}^{\prime}} .
\end{aligned}
$$

\section{LemMa 2.}

$$
\xi^{i k} x_{i j l k}=\frac{1}{2}(n-m)(n-m+1) x_{i k} .
$$

Let $\boldsymbol{i}_{\boldsymbol{r}}$ and $\boldsymbol{k}$, be defined as before. Differentiating every $x_{\boldsymbol{r}}$ in $\boldsymbol{x}_{i j k \boldsymbol{k}}$ and picking out its co-factor one gets

$$
\begin{aligned}
2 \xi_{i k} x_{i j k \boldsymbol{k}}=2\left(\xi_{i k} x_{i k}\right) x_{i \boldsymbol{k}} & +2 \Sigma_{s=1}^{m}\left(\xi^{i k} x_{i k_{s}}\right)(-1)^{s} x_{i k \boldsymbol{\kappa}_{s}} \\
& +2 \Sigma_{r=1}^{m}\left(\xi^{i k} x_{i_{r} k}\right)(-1)^{r} x_{i i_{r} \boldsymbol{k}}+2 \Sigma_{r, s=1}^{m}\left(\xi^{i k} x_{i, k_{k}}\right)(-1)^{r+8} x_{i \boldsymbol{i}_{r} k \boldsymbol{\kappa}_{s}} .
\end{aligned}
$$

Now $2 \xi^{i k} x_{r s}=\delta_{r}^{i} \delta_{s}^{k}+\delta_{s}^{i} \delta_{r}^{k}$, where $\delta_{k}^{i}=0,1$ according as $i \neq k, i=k$. Hence

$$
\begin{aligned}
n(n+1) x_{i \boldsymbol{k}}+(n+1) \Sigma_{\varepsilon=1}^{m} & (-1)^{s} x_{i k_{s} \boldsymbol{k}_{s}}+(n+1) \Sigma_{r=1}^{m}(-1)^{\xi} x_{i_{r} i_{r} \boldsymbol{k}} \\
& +\Sigma_{r, s=1}^{m}(-1)^{r+8} x_{i_{r} \boldsymbol{i}_{r} k_{s} \boldsymbol{k}_{s}}+\Sigma_{r, 8=1}^{m}(-1)^{r+8} x_{k_{s} \boldsymbol{i}_{r} i_{r} \boldsymbol{k}_{s}} .
\end{aligned}
$$

Tbe last term is given by Lemma 1 , the others are plainly multiples of $x_{i k}$. Summing one gets $\left(n(n+1)-2 m(n+1)+m^{2}+m\right) x_{i k}$ $=(n-m)(n-m+1) x_{i k}$, which is the desired result.

THEOREM. If $\dot{\boldsymbol{i}}=i_{1} \ldots i_{m}$ and $\boldsymbol{k}=k_{1} \ldots k_{m}$ then

$$
\xi^{i \boldsymbol{h}} x^{a}=h(\alpha, m) x^{a-1} x^{i k}
$$

where $h(a, m)=\Pi_{k=1}^{m}\left(a+\frac{1}{2}(k-1)\right)$, and $m=1, \ldots n$.

If $\xi_{i k}$ is the algebraical complement of $\xi^{\boldsymbol{k} k}$, an equivalent form of (4) is (5) $\quad \xi_{i k} x^{a}=h(\alpha, n-m) x^{a-1} x_{i k},(m=0, \ldots n-1)$.

When $m=n-1$ one has $\xi_{i k}=\xi^{i k}$ and $\xi^{i k} x^{\alpha}=\alpha x^{a-1}\left(\xi^{i k} x_{r s}\right) x^{r s}=a x^{a-1} x^{i k}$, so that the theorem is true in this case. Now by virtue of (2), $(n-m) \xi_{i k}=\xi^{i k} \xi_{i i k k}$, so that proceeding by induction and using Lemma 2 
Extension of Formula by Cayley to Symmetric Determinants 75 and (3) we have

$$
\begin{aligned}
& (n-m) \xi_{i k} x^{a}=\xi^{i k h} h(a, n-m-1) x_{i j k \boldsymbol{k}} x^{a-1} \\
= & h(a, n-m-1) x^{a-2}\left((a-1) x^{i k} x_{i j k \boldsymbol{k}}+x \xi^{i k} x^{i j k \boldsymbol{k}}\right) \\
= & (n-m) h(a, n-m-1)\left(a-1+\frac{1}{2}(n-m+1)\right) x^{a-1} x_{i \boldsymbol{k}} \\
= & (n-m) h(a, n-m) x^{a-1} x_{i \boldsymbol{k}} .
\end{aligned}
$$

Hence (5) follows for antisymmetrical determinants $x$ and $\xi:(4)$ is valid with a suitable $h(a, m)$ if $m=1$ and $n$ is even and also if $m=n=2$ or 4, but probably in no other cases and certainly not in general.

The Institute of Mathematios,

LUND UNIVERSITY, LOND, SWEDEN. 